\title{
Proper Motion Survey in the Pleiades
}

\section{J. Souchay}

Observatoire de Paris, 61 av. de l'Observatoire, 75014 Paris, France

\author{
E. Schilbach \\ Universität Potsdam/Sternwarte Babelsberg, An der Sternwarte 16, \\ 14482 Potsdam, Germany
}

\begin{abstract}
As a first step of our open cluster programme a catalogue of proper motions and photographic $U, B, V, R$ magnitudes for stars up to $18 \mathrm{mag}$ within a region centered near Alcyone is presented. The catalogue is based on MAMA measurements of plates taken with Tautenburg and OCA (CERGA) Schmidt telescopes. The photometric survey includes ca. 65000 stars and covers a total field of about 25 square degrees. Proper motions have been obtained for ca. 40000 stars within a central 17 square degree region of this field. For the majority of stars in the survey an accuracy of $0.08 \mathrm{mag}$ and $2 \mathrm{mas} /$ year has been estimated for photometric data and proper motions, respectively. The results of the determination of the Pleiades membership up to 18th magnitude is presented.
\end{abstract}

\section{Introduction}

With a distance of $130 \mathrm{pc}$ from the Sun, the Pleiades is one of the most extensively studied open clusters. During recent years much effort has been undertaken to identify faint cluster members (e.g. Stauffer et al. 1991; Hambly et al. 1993). Nevertheless, precise proper motion studies have been mostly restricted to the central field of $1.5^{\circ} \times 1.5^{\circ}$ and to a limiting magnitude of $\mathrm{m}=12$ (Jones 1970; Vasilevskis et al. 1979). The survey discussed here covers a 17 square degree region in the Pleiades and contains proper motions and photographic $U$, $B, V, R$ photometry for ca. 40,000 stars. The accuracy of the data is sufficient to identify cluster members up to $18 \mathrm{mag}$ and, herewith, to improve the basis for theoretical interpretation. According to van Leeuwen (1980), the internal proper motion dispersion of the Pleiades is ca. 1 mas/year. For 45 Pleiades stars in the survey, a proper motion accuracy better than 1 mas/year has been achieved in both components. These stars can be included in the analysis of internal motions in the Pleiades. After the link of proper motions to an extragalactic reference system is established the data for field stars can be used as a test of Galactic models (e.g. Bienaymé et al. 1992; Soubiran 1992, Kharchenko et al. 1994) 


\section{Observations and Measurements}

An essential part of the observations for our proper motion survey is based on plates taken with the Tautenburg Schmidt telescope (134/203/401). Each Tautenburg plate covers $3.3^{\circ} \times 3.3^{\circ}$ of sky with a scale of $51.4 \mathrm{arcsec} / \mathrm{mm} .11$ partly overlapping plate pairs have been used to derive proper motions within a 17 square degree region centered near Alcyone. The epoch difference for the plate pairs ranges from 23 to 30 years and the limiting magnitude from 18 to 20 mag. In addition, three second epoch plates have been included in the programme.

For the photographic $U, B, V, R$ photometry, two plates in each colour taken with the OCA Schmidt telescope (90/152/316) are used. With a size of $5.2^{\circ} \times 5.2^{\circ}$ one OCA plate covers the complete investigated field. A deep plate in $\mathrm{R}$ band taken in 1981 has been selected as a master plate to provide a common identification between stars measured on different plates.

All plates were scanned with the Machine Automatique à Mesurer pour l'Astronomie (MAMA) in its systematic mode with a pixel size of $10 \mu \mathrm{m}$ (Berger et al. 1991)

\section{Proper Motions}

All Tautenburg plates have been transformed to the master plate using 3rd-order polynomials. As a reference, a large sample of anonymous stars (ca. 4,000 stars) has been selected between all field stars with small proper motions and in an intermediate range of magnitudes. For each plate, reduction has been carried out by least square fitting iteratively with a $2.5 \sigma$ criterion. The unit weight error depends on the time baseline between the master plate and a Tautenburg plate and ranges from $1.5 \mu \mathrm{m}$ to $3.0 \mu \mathrm{m}$.

Table 1. Internal accuracy of proper motions and photographic magnitudes in the survey. $N$ is the number of Tautenburg plates used for the determination of proper motions.

\begin{tabular}{|c|c|c|c|c|c|c|c|c|}
\hline $\begin{array}{c}\text { corresponding } \\
\text { magnitude } \\
\text { interval }\end{array}$ & $\begin{array}{r}N \leq 4 \\
\operatorname{rms}(\mathrm{RA}) \\
(\mathrm{mas} / \mathrm{y})\end{array}$ & $\begin{array}{c}N \leq 4 \\
\operatorname{rms}(\mathrm{DE}) \\
(\mathrm{mas} / \mathrm{y})\end{array}$ & $\begin{array}{c}N \geq 20 \\
\operatorname{rms}(\mathrm{RA}) \\
(\operatorname{mas} / \mathrm{y})\end{array}$ & $\begin{array}{c}N \geq 20 \\
\operatorname{rms}(\mathrm{DE}) \\
(\mathrm{mas} / \mathrm{y})\end{array}$ & $\begin{array}{c}U \\
\text { band } \\
\text { (mag) }\end{array}$ & $\begin{array}{c}\text { B } \\
\text { band } \\
\text { (mag) }\end{array}$ & $\begin{array}{c}\mathrm{V} \\
\text { band } \\
\text { (mag) }\end{array}$ & $\begin{array}{c}\mathrm{R} \\
\text { band } \\
\text { (mag) }\end{array}$ \\
\hline$m<9$ & & & 3.9 & 4.2 & 0.10 & 0.06 & 0.10 & 0.11 \\
\hline $9 \leq m<10$ & 4.8 & 6.0 & 1.7 & 1.6 & 0.10 & 0.06 & 0.09 & 0.07 \\
\hline $10 \leq m<11$ & 5.2 & 4.9 & 2.0 & 1.7 & 0.09 & 0.07 & 0.09 & 0.08 \\
\hline $11<m<12$ & 3.5 & 4.1 & 1.4 & 1.1 & 0.08 & 0.05 & 0.08 & 0.08 \\
\hline $12 \overline{<} m<13$ & 3.3 & 3.2 & 1.1 & 1.0 & 0.08 & 0.04 & 0.08 & 0.07 \\
\hline $13 \leq m<14$ & 3.0 & 3.0 & 1.0 & 1.0 & 0.08 & 0.06 & 0.08 & 0.07 \\
\hline $14 \leq m<15$ & 2.7 & 2.9 & 0.9 & 0.9 & 0.08 & 0.07 & 0.08 & 0.07 \\
\hline $15 \leq m<16$ & 2.7 & 2.8 & 1.0 & 1.0 & 0.07 & 0.06 & 0.09 & 0.07 \\
\hline $16 \leq m<17$ & 3.0 & 2.9 & 1.2 & 1.1 & 0.07 & 0.04 & 0.09 & 0.08 \\
\hline $17<m<18$ & 3.8 & 3.6 & 1.7 & 1.6 & 0.09 & 0.06 & 0.11 & 0.09 \\
\hline $18 \leq m<19$ & 5.1 & 4.9 & 2.5 & 2.4 & & 0.09 & 0.13 & 0.10 \\
\hline $19<m$ & 7.0 & 6.6 & & & & 0.11 & & 0.12 \\
\hline
\end{tabular}

Finally, proper motions were obtained by least square adjustment of the transformed positions in dependence on the observational epochs. The proper motion accuracy for stars measured on 2 to 4 Tautenburg plates is compared with 


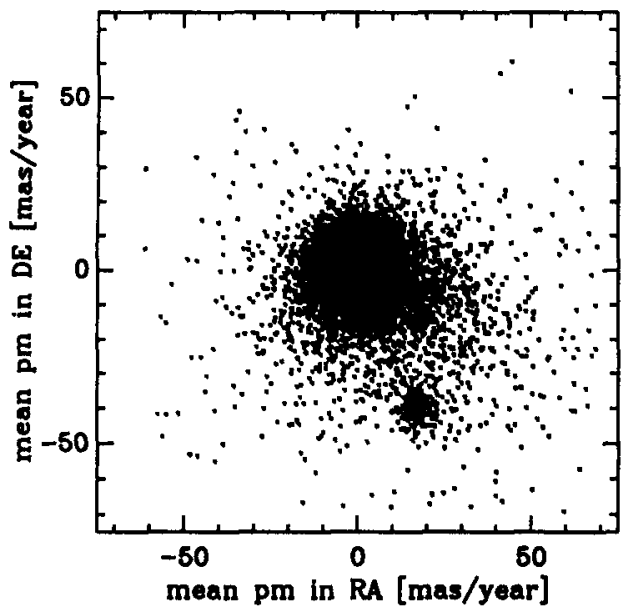

Figure 1. Proper motion diagram for stars brighter than $B=18$ mag.

the positional uncertainties of stars measured on at least 20 Tautenburg plates, as given in Table 1 . The typical uncertainties are between 1 and 2 mas/year.

\section{Photometry}

The photometric calibration of OCA plates was based on sequences taken mostly from Johnson et al. (1958), Landolt (1979) and Stauffer $(1982,1984)$. The correspondence between magnitudes and measured MAMA density fluxes of ca. 370 photometric standards up to 19 mag has been obtained by polynomials containing terms with stellar magnitude, colour and plate coordinates. According to the tests, photometric accuracy can be increased by up to 20 per cent if geometrical local effects are taken into account. The resulting rms ranges from $0.08 \mathrm{mag}$ to $0.15 \mathrm{mag}$. The magnitudes derived on plates of the same colour were averaged. The internal accuracy of photographic $U, B, V, R$ magnitudes obtained from MAMA measurements of OCA plates is given in Table 1.

\section{Survey}

Fig. 1 shows the proper motion diagram for stars up to $B=18 \mathrm{mag}$. The cluster members are clearly separated from the background of field stars and show a strong concentration to the point +17.0 mas/year (RA), -39.3 mas/year (Dec). There are 332 probable Pleiades stars inside a small circle with a radius of about 4 mas/year. Additionally, based on their proper motions, 51 faint $(B<18)$ stars with a proper motion accuracy better than 5 mas/year have been selected as cluster candidates. The mean proper motion errors of 383 stars in the sample are $1.8 \mathrm{mas} /$ year $(\mathrm{RA})$ and $1.6 \mathrm{mas} /$ year $(\mathrm{Dec})$. 
Whereas the majority of cluster members are concentrated around the cluster centre inside an area with a radius of ca. $1.2^{\circ}$, there are some remote candidates in the outer parts of the total field of $3.8^{\circ}(\mathrm{RA}) \times 4.5^{\circ}(\mathrm{Dec})$ examined.

A study of cluster properties including proper motions and UBVR photometry is in progress.

\section{Acknowledgments}

We thank the Tautenburg and OCA Observatories for supplying and the MAMA team for measuring the plates. We are grateful to Jean Guibert and Noël Robichon for helpful discussions. Financial support from PROCOPE (Programme de Cooperation Scientifique between France and Germany) is gratefully acknowledged.

\section{References}

Berger J., Cordoni J. P., Fringant A. M., Guibert J., Moreau O.,Reboul H. \& Vanderriest C., 1991, A\&A, 87, 389

Bienaymé O., Mohan V., Crézé M., Considère S., \& Robin A. C., 1992, A\&A, 253,389

Jones B. F., 1970, AJ, 75, 563

Johnson H. L. \& Mitchell R. I., 1958, AJ, 128, 31

Hambly N. C., Hawkins M. R. S. \& Jameson R. F., 1993, A\&AS, 100, 607

Kharchenko, N., Schilbach, E. \& Scholz, R.-D., 1994, Astron.Nachr., 315, in press

Landolt A. U., 1979, AJ, 231, 468

Soubiran C., 1992, A\&A, 259, 394

Stauffer J., 1982, AJ, 87, 1507

Stauffer J., 1984, ApJ, 280, 189

Stauffer J., Klemola, A., Prosser, C. \& Probst R., 1991, AJ, 101, 980

van Leeuwen F., 1980, in Star Clusters, J. E. Hesser, ed., (Reidel, Dordrecht)

Vasileuskis S., van Leeuwen F., Nicholson W. \& Murray C. A., 1979 A\&AS, 37, 333

\section{Discussion}

Szécsényi-Nagy: According to one of your viewgraphs, your programme stars range from $B=8$ to $B=19$. Could you say something about the distribution in brightness of the Pleiades member stars. Could you give the approximate number of cluster member which are fainter than $B=16$ ?

Schilbach: According to our first analysis of the sample, there are 150 Pleiades candidates with $B>16$, for a total number of 380 stars for which the membership probability is very high $(>90 \%)$. 